\title{
Addressing future challenges for cancer services: part I
}

Jane Maher ${ }^{* 1}$ \& Gina Radford ${ }^{2}$

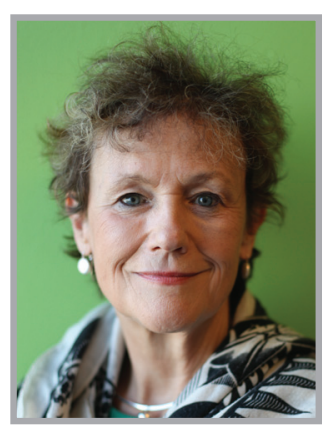

Jane Maher \& Gina Radford speak to Gemma Westcott, Commissioning Editor

Jane Maher has been Macmillan's Chief Medical Officer since 1999 and now shares the role as Joint Chief Medical Officer with general practitioner Rosie Loftus, reflecting the growing need for specialists and generalists to work more effectively together. She has been a National Health Service (NHS) Improvement Clinical Leader for over 10 years and is a Consultant Clinical Oncologist at Mount Vernon Cancer Centre where she has worked for more than 20 years, during which she helped develop nonsurgical oncology services in five district general hospitals. Jane chaired the Maher Committee for the Department of Health in 1995, led the UK National Audit of Late Effects Pelvic Radiotherapy for the Royal College Of Radiologists (RCR) in 2000 and, most recently, chaired the 'National Cancer Survivorship Initiative, consequences of treatment work stream'. She co-founded one of the first Cancer Support and Information services in the UK, winning the Nye Bevan award in 1992 and there are now more than 60 units based on this model. She is a member of the Older People and Cancer Clinical Advisory Group. She has written more than 100 published articles and is a UK representative for cancer survivorship in Europe and advises on Cancer survivorship programs in Denmark and Canada.

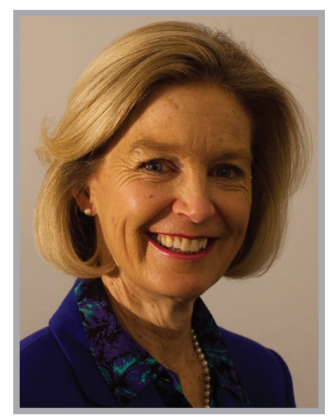

Gina Radford is Deputy Chief Medical Officer for England, a post she took up in January 2015. Prior to that, she has held a number of roles in public health, at local and regional level. Most recently she was as Centre Director for Anglia and Essex for Public Health England, and as part of that role helped lead nationally on the public health response to Ebola. She was until very recently Chair of one of the NICE public health advisory committees. She has previously worked on a number of national projects, including leading the Department of Health's response to the Shipman Enquiry, undertaking a review of specialist public health for CMO Scotland, chairing a national short life working group looking at the issue of making difficult decisions in NHS Scotland, and undertaking the evaluation of the first pilot (regional bowel cancer detection pilot) for the Be Clear on Cancer National Awareness and Early Diagnosis campaign, on behalf of the Department of Health and Cancer Research UK. Outside work, Gina is a Licensed Lay Minister in the Church of England, and is training to be ordained. She enjoys riding, walking the somewhat aging dog, reading and is the village duck warden!

'Macmillan Cancer Support, 89 Albert Embankment, London, SE1 7UQ, UK

2Department of Health, 79 Whitehall, SW1A 2NS, UK

*Author for correspondence: jmaher@macmillan.org.uk

Future
Medicine $\%$ part of 


\section{KEYWORDS}

- bowel cancer screening

- cancer services • cancer strategy for England - cost of cancer $\bullet$ Independent Cancer Taskforce • National Cancer Survivorship Initiative $\bullet$ patient priorities $\bullet$ public health - survivorship

First draft submitted: 24 November 2015; Accepted for publication: 1 December 2015; Published online: 5 February 2016

Q Could you both tell our readers about where your interest in health policy stemmed from?

\section{- Gina Radford}

Having trained as a GP, I thoroughly enjoyed my clinical work and meeting with patients and their families. But too often I was being asked to do things too late, or I saw diseases and situations that could have been prevented. So I decided to change specialty and trained in public health. I wanted to try to prevent some of the conditions I had seen as a GP, and this meant looking at what we need to do on a population basis, not just an individual patient basis. Part of this is the policies we have for and influencing health. I first became closely involved in health policy personally when I was a Director of Public Health, having to respond to the challenges of how to afford some of the new drugs with finite resources. This was before National Institute for Health and Care Excellence existed, so each District Health Authority had to make their own decisions, based on evidence that public health teams often provided. It was the challenge of making these difficult decisions that really got me thinking about what health policy means in practice, and from there my interest and involvement grew.

\section{- Jane Maher}

When I first started as a consultant oncologist, I asked a group of patients how I should spend the next 30 years of my career. They said if I could not cure cancer, could I help to get information, support and communication right (both between patient and professionals and between all the different professionals looking after them)? That conversation had a big impact on the focus of my research, which until then had been conventional translational research. I worked through the conventional academic and college hierarchy, but it was not until I started working with a big charity that I realized the importance of influencing overarching policy and how evidence on its own is not enough to make change happen.

What has been your favorite moment in your careers so far?

- Gina Radford

I could not pick one moment - I have had so many really positive experiences in my career. When I look back on my career I have to say, there was not any career plan! So it never ceases to amaze me that I am where I am today.

So there is no one moment - rather a large collection of wonderful memories that I bring out every now and then to enjoy and spur me on.

The best memories tend to be about the people I have met. Most have taught me an awful lot. I remember meeting the most wonderful transgender group when I was a naive young Doctor of Public Health, who taught me that taking time to find out about peoples' experiences and views is vital. I spent quite a bit of my junior doctor years working with children, and I loved working with neonates. They taught me about the tenacity of the human spirit, and the wonder that is a new living person.

I have had a career that I have thoroughly enjoyed, and I know I am lucky. I love my current job, just as to be honest I have loved most jobs. I can honestly say, without wishing to sound horribly soft, that there are not too many other careers that I would have wanted!

\section{- Jane Maher}

There have been so many moments but perhaps my favorite was when a young woman who I had treated as a child early in my career for leukemia brought me a bottle of champagne when she got into medical school!

Q How would you describe the current state of cancer services in the UK?

\section{- Gina Radford}

Survival rates are at a record high despite the NHS treating more people for cancer than ever before. For people diagnosed from 2011 to 2015 , we are on track to save a projected 12,000 extra lives a year by the end of 2015 . This is over double our ambition of 5000 as set in the 2011 Cancer Outcomes Strategy.

Of course there is more to do, and the aim is for us to become the best in the world for cancer care and outcomes. We welcome the Independent Cancer Taskforce's new cancer strategy, which was published to wide acclaim on 19 July. It recommends improvements across the cancer pathway and we are working with the NHS, charities and patient groups to deliver it. 


\section{- Jane Maher}

This is a time of great challenge but also great opportunity. Survival rates are increasing but we know that many people who do survive are not living in good health - indeed at least one in four survivors have to deal with the consequences of cancer and its treatment. Services have not yet grasped the challenge of providing better support to either these patients, or those with cancer as well as other co-morbidities. Although half of cancer patients will live more than a decade after diagnosis, many do not receive the rehabilitation they need.

The continued breach of the 62-day cancer waiting time target across the UK highlights the pressure that services and healthcare professionals are under just to deliver timely diagnosis and treatment. Yet the benefits of improved survival will not be realized unless aftercare receives more attention to ensure people can return to work, change their lifestyles and, particularly for the elderly, maintain independence after cancer treatment.

\section{Q What trends are we seeing in cancer} epidemiology \& should we be worried about a widening survivor community?

\section{- Jane Maher}

We know that there are currently 2.5 million people living with cancer in the UK and this will rise to at least 4 million by 2030 . The increase in prevalence is down to several factors, namely a growing and aging population leading to a rise in incidence, coupled with improvements in diagnosis and treatment. However, we know that many people who survive long term do not live in good health, even with cancer types that have relatively high survival rates, such as breast cancer. Just over $50 \%$ of people living with cancer have one or more moderate or severe unmet needs, such as depression, anxiety or tiredness, 6 months after the end of treatment, and at least one in four people face poor long-term health or disability. We also know that more than two in three people with cancer are also living with one or more other potentially serious long-term conditions, and this proportion will rise as the population continues to age. Another trend is the emergence of a distinct group of people with incurable but treatable cancer. We need to recognize and respond to the changing nature of cancer as a matter of urgency.

\section{- Gina Radford}

As Jane said, we know that the number of cancer survivors continues to grow. The National
Cancer Survivorship Initiative has recommended four priority areas: recovery packages; consequences of treatment and patient-reported outcome measures; stratified pathways of care and physical activity. To support this work, NHS England, in partnership with Macmillan Cancer Support, has established the Living with and Beyond Cancer Program, a 2-year program of work to embed the four priority areas from National Cancer Survivorship Initiative into mainstream commissioning.

Q There is a clear focus for work to prevent cancer \& improve cancer services. What are the major recent developments in your areas of work?

\section{- Jane Maher}

The publication of the Cancer Strategy for England has been a major milestone for cancer this year. The Strategy's six strategic priorities include recommendations relating to patient experience, transforming care and support for people living with and beyond cancer, the cancer workforce and the commissioning process. Macmillan is part of the Independent Cancer Taskforce that developed the recommendations in the Strategy, and we are pleased that it reflects the need to think beyond diagnosis, treatment and survival, essential though these are. Only if the Strategy is fully funded and implemented can it ensure that cancer services are imporved across the pathway.

Macmillan is also working to ensure cancer remains a priority for the next governments in Scotland, Wales and Northern Ireland. It is vital that we implement cancer plans and strategies across the UK - and benchmark them against each other's best practice.

\section{- Gina Radford}

As Jane mentioned, the strategy proposes six strategic priorities over the next 5 years.

The first of these is 'spearheading a radical upgrade in prevention and public health', and identifies a new tobacco control strategy and a national action plan on obesity as key areas where the NHS can work with government to drive improvements in public health.

The second priority centers on 'driving a national ambition to achieve earlier diagnosis'. There is work already ongoing in this regard and we will seek to continue to improve. NICE recently updated referral guidelines for suspected cancer which could save about 5000 lives by 
encouraging GPs to think of cancer sooner and lower the threshold at which people are given cancer tests.

Elsewhere, NHS England launched a new program to test innovative ways of diagnosing cancer earlier, and we have run numerous national 'Be Clear on Cancer' awareness campaigns, including a national reminder campaign on breast cancer in women over 70 years which started on 13 July and ran till 6 September 2015.

The NHS Bowel Cancer Screening Program in England has introduced a new screening test, where both men and women are invited for 'bowel scope screening' around the time of their 55th birthday. Bowel scope screening is an examination called 'flexible sigmoidoscopy' which looks inside the lower bowel. The aim is to prevent bowel cancer by finding and treating any polyps which may develop into bowel cancer if left untreated. Bowel scope screening is an addition to the existing NHS Bowel Cancer Screening Program, and is a one off examination which will play a significant role in preventing bowel cancer. The pilot was carried out in six sites. Progress is on course to meet the Secretary of State for Health's commitment to have $100 \%$ of centers being live by the end of 2016 .

Q What do you believe are patients' priorities for cancer care?

- Gina Radford

The Taskforce's new strategy sets out a vision for what cancer patients should expect from the health service: effective prevention (so that people do not get cancer at all if possible); prompt and accurate diagnosis; informed choice and convenient care; access to the best effective treatments with minimal side effects; always knowing what is going on and why; holistic support; and the best possible quality of life, including at the end of life. The third strategic priority centers on: 'Establishing patient experience as being on a par with clinical effectiveness and safety'.

It is important for us to ensure that people with cancer have the best possible experience throughout the cancer pathway and are treated with dignity and respect. We were pleased to see that the results of the 2014 Cancer Patient Experience Survey published in September, showed some improvement on many of the scores since the last survey $-89 \%$ of patients reported that their care was either excellent or very good. We expect the next survey to be launched later this year, with the results available in 2016 .
It is quite likely that patient expectations will increase in coming years, so that maintaining or improving on this level of satisfaction will require considerable effort. Some patient groups, such as those with rare and less common cancers, report less satisfactory experience. In addition, some areas of the country score poorly on patient satisfaction measures and we should expect these areas to deliver significant improvement in the coming years. The cancer strategy notes that Cancer Patient Experience Survey should continue to evolve and should be repeated every year, with patient satisfaction measured for every hospital and Clinical Commissioning Group.

\section{- Jane Maher}

Of course patients want to be cured of cancer, but every person with cancer is an individual with their own needs and priorities. However, we have worked with patients to develop a set of statements - our 'Nine Outcomes' - that provide a broad overview of their priorities, including 'I am treated with dignity and respect' and 'I can enjoy life' as well as 'I get the treatment and care that is best for my cancer and my life'. Together the statements cover early diagnosis, patient experience, appropriate care and treatment, support for carers, quality of life and end-of-life care.

We also have a good understanding of people's common concerns, from our electronic holistic needs assessment tool. Worry, fear or anxiety, tiredness/fatigue, sleep problems/nightmares and pain are all common. We also know many patients with pelvic cancers have impaired bowel control. A simple holistic needs assessment tool can help both healthcare professionals and patients to identify people's key concerns, have a more focused conversation and develop a realistic plan to address their needs.

\section{Q What do you think are the biggest} challenges we face \& how can we prepare our cancer services for a changing future?

\section{- Jane Maher}

In addition to the challenges posed by the change in epidemiology trends, economics is another major area for concern. The cost of cancer to the NHS has been a challenge for some time, but the issue is becoming seriously acute. In England, the current annual cost of cancer services is at least $\mathrm{GB} £ 6.7$ billion and predicted to rise to GB£13 billion by 2020 . We are calling on the government to continue to 
fund cancer services at the current growing rate, to front-load investments to realize efficiencies sooner and to fund the implementation of the Cancer Strategy for England with an additional GB£400 million per year over the next 5 years. Similar challenges exist elsewhere in the UK, where shrinking budgets mean the health and social care system is under huge pressure to deliver, and the proportion of older people in the population is higher. It is vital that investment in cancer services continues to grow to meet the demand placed on both health and social care budgets.

A second big challenge is the availability of workforce. We know that there is likely to be a shortage of over a million health professionals overall in the EU by 2020 and that many experienced staff are leaving the professions or retiring early. There has been a movement to specialization over the last few decades but specialist teams communicate less and less with each other and there is a loss of generalist skills, in both hospitals and the community. We have to develop new ways of working and ensure specialist skills are shared more effectively.

\section{- Gina Radford}

In addition to the Cancer Taskforce report's strategic priorities mentioned earlier, the remaining three are:

- Transforming our approach to support people living with and beyond cancer;

- Making the necessary investments required to deliver a modern high-quality service; and

- Overhauling processes for commissioning, accountability and provision.

There has been a great deal done in recent years to improve cancer outcomes, and the new strategy, which will take us through to 2020, recommends improvements in all areas for us to continue to do better. The gap in survival rates still remains between England and the best performing countries - therefore this is one of the key challenges we face.

The number of people diagnosed, and living, with cancer each year will continue to grow rapidly, even with major improvements in prevention. The primary reasons for this are our aging population and our success in increasing survival. This will place significant additional demand on health and social care services.

Cancer survival in England has improved significantly over the last 15 years. More than half of people receiving a cancer diagnosis will now live 10 years or more. But our mortality rates are higher than they could be. In addition, variability exists in access to, and experience of, care across different areas, subgroups of the population and cancer types. There is much we could do to improve patient experience and longterm quality of life, and to make our care more patient-centered.

\section{Acknowledgements}

Professor Jane Maher thanks colleagues at Macmillan for their helpful input into this article.

\section{Disclaimer}

The opinions expressed in this article are those of the authors and do not necessarily reflect the views of Future Medicine Ltd.

\section{Financial \& competing interests disclosure}

$J$ Maher and $G$ Radford have no relevant affliations or financial involvement with any organization or entity with a financial interest in or financial conflict with the subject matter or materials discussed in the manuscript. This includes employment, consultancies, honoraria, stock ownership or options, expert testimony, grants or patents received or pending, or royalties.

No writing assistance was utilized in the production of manuscript. 